\title{
Efecto del extracto de cramberry sobre la capacidad adhesiva de la placa dental en dientes de niños de 10 años del colegio Horacio Morales Delgado. Arequipa-Peru 2016.
}

Effect of cranberry extract on the adhesive capacity of dental plaque in the teeth of 10-year-old children from the Horacio Morales Delgado school. Arequipa-Peru 2016.

Andy Paucar Rodriguez y Rufo Figueroa-Banda

Universidad Católica de Santa María. Arequipa Perú.

\section{INFORMACIÓN}

Historia del Artículo

Recepción: 18/11/2019

Revisión: 25/01/2020

Aceptación: 20/05/2020

\section{Palabras Clave}

Capacidad adhesiva, extracto de Cranberry, placa dental, índice de placa dental.

\section{Key Words}

Adhesive capacity, Cranberry extract, dental plaque, dental plaque index

\section{DOI}

https://doi.org/10.35286/veritas. v21i2.283

\begin{abstract}
RESUMEN
OBJETIVOS: Los objetivos de la presente investigación fue determinar el efecto del cranberry sobre la capacidad adhesiva de la placa dental en los niños del colegio Horacio Morales de la ciudad de Arequipa Perú. METODOLOGIA Y METODOS: Para esta investigación se probó el extracto de cranberry en 17 niños de 10 años del colegio Horacio Morales Delgado de Arequipa Perú. Esta investigación tuvo una duración de dos días, el primer día se procedió al cepillado de dientes a los niños los cuales permanecieron durante una hora sin ingesta de alimentos, al término de la cual se les tomo el índice de placa dental (grupo control). El segundo día se procedió también al cepillado de dientes a los niños e inmediatamente se les aplico el extracto de Cranberry como un colutorio, después de una hora sin haber ingerido ningún alimento ni bebidas, se les tomo el índice de placa dental. RESULTADOS: Los resultados obtenidos de la evaluación fueron medidos con el índice de placa dental el cual tiene valores de $0,1,2$, 3; dependiendo de cuanta placa se encuentre en la pieza dental (Índice de placa de Silness y Loe). El índice de placa dental promedio en los dientes de niños no tratados con el extracto de Cranberry y solo cepillados con pasta profiláctica fue 1.18. El índice de placa dental promedio en los dientes de niños tratados con el extracto de Cranberry fue de 0.18 . Los resultados evidencian un contraste muy significativo en los dos días del experimento, llegando a la conclusión que usando el extracto de Cranberry reduce notoriamente el nivel de placa dental adherida al esmalte de las piezas dentales.
\end{abstract}

\begin{abstract}
OBJECTIVES: The objectives of this research were to determine the effect of the cranberry on the adhesive capacity of dental plaque in the children of the Horacio Morales school in the city of Arequipa, Peru. METHODOLOGY AND METHODS: For this research, cranberry extract was tested on 17 10-year-old children at the Horacio Morales Delgado School in Arequipa, Peru. This research lasted two days, the first day we proceeded to brush teeth to children who remained for an hour without food intake, at the end of which they were taken the dental plaque index (control group). On the second day we also proceeded to brush the children's teeth and immediately applied the Cranberry extract as a mouthwash, after an hour without having ingested any food or drink, we took the dental plaque index. RESULTS: The results obtained from the evaluation were measured with the dental plaque index which has values of $0,1,2,3$; depending on how much plaque is in the dental piece (Silness and Loe plaque index). The average plaque index in teeth of children not treated with Cranberry extract and only brushed with prophylactic paste was 1.18 . The average plaque index in teeth of children treated with Cranberry extract was 0.18 . The results show a very significant contrast in the two days of the experiment, reaching the conclusion that using Cranberry extract significantly reduces the level of dental plaque adhered to the enamel of the teeth.
\end{abstract}

\section{INTRODUCCIÓN}

La cavidad oral humana (boca) alberga un microbioma complejo que consiste en bacterias, archaea, protozoos, hongos y virus. Estas bacterias son responsables de dos enfermedades comunes de la boca humana, incluyendo la periodontitis (encías) y la caries dental (caries). Las caries

Correspondencia:

Rufo Alberto Figueroa Banda

rufofigueroa@hotmail.com dentales son causadas por placas, que son una comunidad de microorganismos. (1) La caries dental se ha considerado como la enfermedad de mayor peso en la historia de la morbilidad bucal a nivel mundial. En la actualidad, su aparición se asocia en gran manera con factores socioculturales, económicos, del ambiente y del comportamiento. Afecta entre el $60 \%$ y $90 \%$ de la población escolar según la OMS(2). Es por esto que se necesita prestarle la debida atención a la prevención de estas enfermedades que causan tanto perjuicio en la salud de las personas.

La enfermedad periodontal es la segunda causa de morbilidad bucal no solo en los países industrializados sino en los que están en vía de desarrollo (3). La enfermedad 
periodontal es considerada una enfermedad infecciosa inflamatoria que de acuerdo al grado de compromiso puede llevar a la pérdida total de los tejidos de soporte de los dientes(4). La gingivitis es considerada una forma inicial de la enfermedad periodontal, puede convertirse en una periodontitis, incrementando su severidad en base a la combinación de factores como la falta de cepillado(5). Es por eso el afán de la comunidad odontológica de tratar de combatirla.

En los niños las enfermedades del periodonto se clasifican en cuatro grupos: aquellas alteraciones periodontales, que son manifestaciones de padecimientos sistémicos, en las cuales no se observan factores locales responsables; la enfermedad periodontal necrotizante asociada a disminución en la respuesta inmune de los tejidos periodontales a los productos bacterianos y que manifiesta en casos de inmunosupresión como la desnutrición; la periodontitis destructiva agresiva, caracterizada por una rápida pérdida de los tejidos periodontales, presente principalmente en la etapa de la pubertad y la enfermedad periodontal crónica de mayor prevalencia que las anteriores, menos agresiva y más localizada en la zona gingival, provocada por factores locales como la acumulación de placa dentobacteriana por una higiene deficiente (6).

Existen bastantes microorganismos como Streptococcus mutans, Streptococcus sobrinus y Lactobacillus spp., entre otros, contribuyen a la formación de biopelículas dentales (7). Streptococcus mutans es uno de los microorganismos cariogénicos asociados a la caries dental. De acuerdo con la hipótesis de la placa ecológica, la caries dental es la consecuencia de cambios en el balance natural de la microflora de la placa dental causados por la alteración de las condiciones ambientales locales (homeostasis microbiana oral (8). S. Sobrinus ha sido implicado en el desarrollo de caries, particularmente en casos donde el desarrollo de caries parece ser independiente de S. Mutans. Es interesante que S. Sobrinus muestre una mayor producción de ácidos y tolerancia a los ácidos en comparación con S. Mutans

El cranberry (vaccinium macrocarpon) se ha utilizado durante cientos de años como fuente de alimento, y ha sido reconocidos por las propiedades para la salud que poseen. Los nativos americanos lo usaban para dar sabor y conservar la carne y también como medicina (9). El concentrado de arándano americano (V. macrocarpon) tiene un alto poder de atracción. Efectos antibacterianos contra los patógenos transmitidos por los alimentos como : E. coli O157:H7, L. monocytogenes, S. Typhimurium y S. aureus in vitro (10).

El arándano ( Vaccinium macrocarpon ), una fruta medicinal natural, se ha utilizado ampliamente como un suplemento dietético botánico popular, especialmente en forma de extracto de arándano, para prevenir y tratar las infecciones del tracto urinario causadas por UPEC (Escherichia coli uro patógena) (11). Esta fruta es muy usada para combatir este tipo de infecciones urinarias por los diferentes compuestos que el cranberry posee. En investigaciones médicas, de caries dental y enfermedad periodontal, se han verificado los beneficios relacionados con los polifenoles de Cranberrys. El extracto de arándano tiene la capacidad de inhibir la adhesión deS.sobrinusa la dentina y reducir el desarrollo de caries dental "in vivo! Además, informaron que el Cranberry pudo inhibir la producción de mataloproteinasas de matriz en tejidos periodontales inflamados y la actividad catalítica de mataloproteinasas de matriz-1 y metaloproteinasas de matriz-9 (12).

La adherencia de la placa a la superficie gingival es otro problema que es recurrente en la población infantil ya que sabemos que para eliminar esta capa se necesitan de métodos físicos y químicos que muchas veces no son bien utilizados por la población y mucho menos por los niños. La inhibición de la adhesión es un objetivo atractivo para el desarrollo de nuevas terapias en la prevención de infecciones bacterianas, particularmente infecciones de las superficies mucosas.P. gingivalis. El hecho de que el extracto de cranberry actúa previniendo la adhesión bacteriana en lugar de inhibir el crecimiento puede representar una ventaja ya que las bacterias no pueden desarrollar resistencia. (13)

Hay varios compuestos encontrados en el cranberry que tienen beneficios para la salud. Las antocianidinas, las proantocianidinas y los flavonoides son tres de los compuestos del cranberry con posibles beneficios para la salud. Las antocianidinas y las proantocianidinas sirven para proteger la planta de infecciones a través de propiedades antiadherencia.(14).

Las antocianinas son un grupo de pigmentos de color rojo, hidrosolubles, ampliamente distribuidos en el reino vegetal. Químicamente las antocianinas son glicósidos de las antocianidina. Los extractos crudos de arándano, en varios vehículos, han mostrado efectos inhibitorios sobre la formación de biopelículas orales in vitro. La presencia de proantocianidinas (PAC) en los extractos de arándano se ha relacionado con actividades biológicas contra los atributos de virulencia específicos de Streptococcus mutans, por ejemplo, la inhibición de la actividad glucosiltransferasa $(\mathrm{Gtf})(14)$.

\section{MATERIALES Y MÉTODOS}

\section{Identificación de la Técnica}

Esta fue una investigación cuantitativa, experimental, prospectiva, descriptiva, comparativa y de campo en la cual estudiamos la capacidad antiadherente del extracto de Cranberry sobre los dientes.

\section{Técnica}

Se eligió a niños que cumplan los requerimientos de inclusión para aplicarles el colutorio de arándano del aula de 5to grado de primaria de la INSTITUCION EDUCATIVA. Horacio Morales Delgado, Arequipa-Perú.

La prueba se aplicó en dos días; el primer día se realizó higiene bucal de los niños con pasta profiláctica después de una hora se les controlo con una pastilla reveladora y se tomó los datos referentes al nivel de placa dental.

El segundo día hicimos el mismo procedimiento de higiene oral con pasta profiláctica pero después de esto aplicamos el colutorio de Cranberry, después de una hora hicimos el control con pastillas reveladoras y también tomamos datos del nivel de placa dental.

Los datos se registraron en una ficha, tomando el rango de: 0-1 -2-3; (índice de SILLNES). 


\section{Control de grupos}

Criterios de inclusión y exclusión:

A. Criterios de inclusión: Niños del 5to grado de primaria de la institución educativa Horacio Morales Delgado que tengan 10 años.

B. Criterios de exclusión: Niños del 5to grado de primaria de la institución educativa Horacio Morales Delgado que no tengan 10 años, O QUE NO HAYAN TRAIDO CONSENTIMIENTO INFORMADO

\section{RESULTADOS}

\section{Procesamiento y análisis de los resultados}

Tabla 1: Índice de placa dental en los alumnos después del uso del extracto de Cranberry. Fuente: Matriz de datos

\begin{tabular}{|c|c|c|c|c|c|c|}
\hline \multirow{3}{*}{$\begin{array}{c}\text { IPD } \\
\text { Con colutorio de extracto } \\
\text { de Cranberry }\end{array}$} & \multicolumn{4}{|c|}{ Arcada } & \multirow{2}{*}{\multicolumn{2}{|c|}{ Total }} \\
\hline & \multicolumn{2}{|c|}{ Superior } & \multicolumn{2}{|c|}{ Inferior } & & \\
\hline & $\mathrm{N}^{\circ}$ & $\%$ & $\mathrm{~N}^{\circ}$ & $\%$ & $\mathrm{~N}^{\circ}$ & $\%$ \\
\hline Exento de placa & 13 & 76.5 & 14 & 82.4 & 27 & 79.4 \\
\hline Índice de placa 1 & 3 & 17.6 & 3 & 17.6 & 6 & 17.6 \\
\hline Índice de placa 2 & 1 & 5.9 & 0 & 0.0 & 1 & 2.9 \\
\hline Índice de placa 3 & 0 & 0.0 & 0 & 0.0 & 0 & 0.0 \\
\hline $\mathrm{P}$ & \multicolumn{6}{|c|}{$0.595(\mathrm{P} \geq 0.05)$ N.S. } \\
\hline Media Aritmética & \multicolumn{2}{|c|}{0.29} & \multicolumn{2}{|c|}{0.18} & \multicolumn{2}{|c|}{0.24} \\
\hline Desviación Estándar & \multicolumn{2}{|c|}{0.58} & \multicolumn{2}{|c|}{0.39} & \multicolumn{2}{|c|}{0.49} \\
\hline IPD Mínimo & \multicolumn{2}{|c|}{0} & \multicolumn{2}{|c|}{0} & \multicolumn{2}{|c|}{0} \\
\hline IPD Máximo & \multicolumn{2}{|c|}{2} & \multicolumn{2}{|c|}{1} & \multicolumn{2}{|c|}{2} \\
\hline $\mathrm{P}$ & \multicolumn{6}{|c|}{$0.498(\mathrm{P} \geq 0.05)$ N.S. } \\
\hline Total & 17 & 100.0 & 17 & 100.0 & 34 & 100.0 \\
\hline
\end{tabular}

PRUEBAS USADAS: Main whitney - T de Student

Vemos que en la arcada superior la mayoría de los pacientes $76.5 \%$ están exentos de placa y en la arcada inferior también la mayoría el $82.4 \%$ también están exentos de placa dental. Según la prueba estadística no hay diferencia significativa, eso quiere decir que tanto en superior como inferior no presenta placa blanda.

Desde el punto de vista cuantitativo el promedio del índice de placa dental de la arcada superior es de 0.29 y el de la arcada inferior es de 0.18 , considerándolo un valor no significativo.

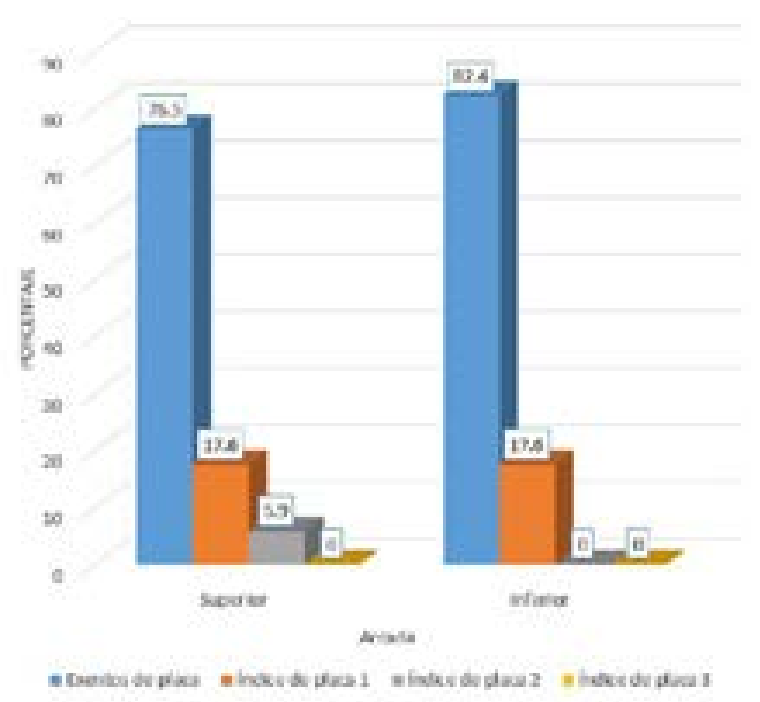

Gráfico 1: Índice de placa dental en los alumnos después del uso del extracto de Cranberry. Fuente: Matriz de datos.

Tabla 2: Comparación del índice de placa dental en la arcada superior en los dientes de los alumnos antes y después del uso del extracto de Cranberry. Fuente: Matriz de datos

\begin{tabular}{|c|c|c|c|c|}
\hline \multirow{3}{*}{$\begin{array}{c}\text { IPD } \\
\text { Arcada Superior }\end{array}$} & \multicolumn{4}{|c|}{ Grupo } \\
\hline & \multicolumn{2}{|c|}{ Sin Cranberry } & \multicolumn{2}{|c|}{ Con Cranberry } \\
\hline & $\mathrm{N}^{\circ}$ & $\%$ & $\mathrm{~N}^{\circ}$ & $\%$ \\
\hline Exentos de placa & 0 & 0.0 & 13 & 76.5 \\
\hline Índice de placa 1 & 7 & 41.2 & 3 & 17.6 \\
\hline Índice de placa 2 & 8 & 47.1 & 1 & 5.9 \\
\hline Índice de placa 3 & 2 & 11.8 & 0 & 0.0 \\
\hline $\mathrm{P}$ & \multicolumn{4}{|c|}{$0.000(\mathrm{P}<0.05)$ S.S. } \\
\hline Media Aritmética & \multicolumn{2}{|c|}{1.71} & \multicolumn{2}{|c|}{0.29} \\
\hline Desviación Estándar & \multicolumn{2}{|c|}{0.68} & \multicolumn{2}{|c|}{0.58} \\
\hline IPD Mínimo & \multicolumn{2}{|c|}{1} & \multicolumn{2}{|c|}{0} \\
\hline IPD Máximo & \multicolumn{2}{|c|}{3} & \multicolumn{2}{|r|}{2} \\
\hline $\mathrm{P}$ & \multicolumn{4}{|c|}{$0.000(\mathrm{P}<0.05)$ S.S. } \\
\hline Total & 17 & 100.0 & 17 & 100.0 \\
\hline
\end{tabular}

Vemos que en los alumnos antes del uso del extracto la mayoría $47.1 \%$ se presentó placa en índice 2 y después del uso del extracto de Cranberry la gran mayoría $76.5 \%$ están exentos de placa, según la prueba estadística estas diferencias si son significativas, por lo tanto, el uso del extracto de Cranberry determina que haya menos adhesión de placa en los dientes de la arcada superior. 


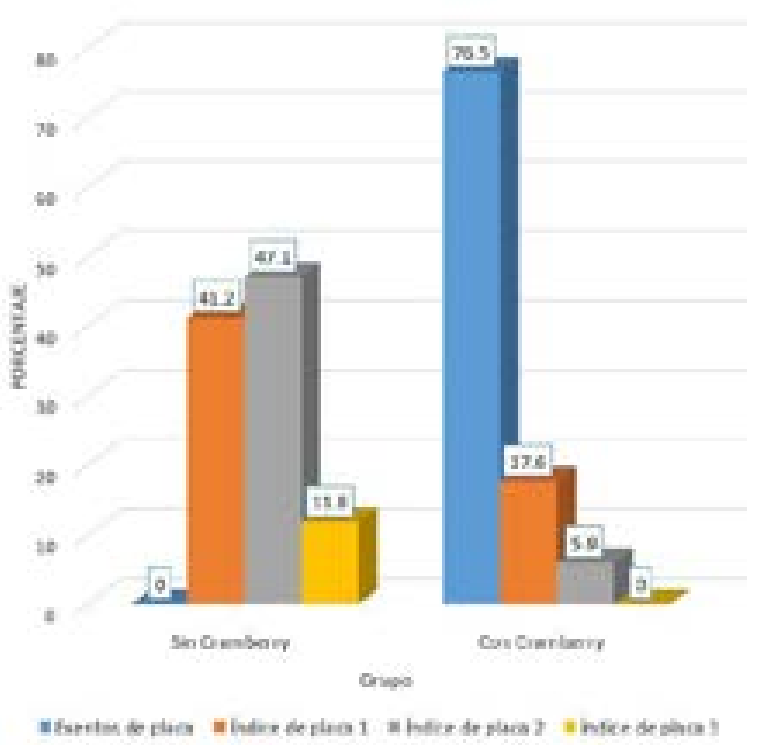

Gráfico 2: Comparación del índice de placa dental en la arcada superior en los dientes de los alumnos antes y después del uso del extracto de Cranberry. Fuente: Matriz de datos

Tabla 3: Comparación del índice de placa dental en la arcada inferior en los dientes de los alumnos antes y después del uso del extracto de Cranberry. Fuente: Matriz de datos

\begin{tabular}{|c|c|c|c|c|}
\hline \multirow{3}{*}{$\begin{array}{c}\text { IPD } \\
\text { Arcada Inferior }\end{array}$} & \multicolumn{4}{|c|}{ Grupo } \\
\hline & \multicolumn{2}{|c|}{ Sin Cranberry } & \multicolumn{2}{|c|}{ Con Cranberry } \\
\hline & $\mathrm{N}^{\circ}$ & $\%$ & $\mathrm{~N}^{\circ}$ & $\%$ \\
\hline Exentos de placa & 2 & 11.8 & 14 & 82.4 \\
\hline Índice de placa 1 & 12 & 70.6 & 3 & 17.6 \\
\hline Índice de placa 2 & 1 & 5.9 & 0 & 0.0 \\
\hline Índice de placa 3 & 2 & 11.8 & 0 & 0.0 \\
\hline $\mathrm{P}$ & \multicolumn{4}{|c|}{$0.001(\mathrm{P}<0.05)$ S.S. } \\
\hline Media Aritmética & \multicolumn{2}{|c|}{1.18} & \multicolumn{2}{|c|}{0.18} \\
\hline Desviación Estándar & \multicolumn{2}{|c|}{0.80} & \multicolumn{2}{|c|}{0.39} \\
\hline IPD Mínimo & \multicolumn{2}{|c|}{0} & \multicolumn{2}{|r|}{0} \\
\hline IPD Máximo & \multicolumn{2}{|c|}{3} & \multicolumn{2}{|r|}{1} \\
\hline $\mathrm{P}$ & \multicolumn{4}{|c|}{$0.000(\mathrm{P}<0.05)$ S.S. } \\
\hline Total & 17 & 100.0 & 17 & 100.0 \\
\hline
\end{tabular}

Observamos que en los alumnos antes del usó del extracto de Cranberry la mayoría $70.6 \%$ presento placa con índice 1 y después del uso del extracto de Cranberry estuvieron exentos de placa un $82.4 \%$.

Desde el punto de vista cuantitativo el promedio del índice de placa dental antes del uso del colutorio es de 1.18 y el promedio después del uso del extracto de Cranberry es de 0.18 , estos valores si son significativos, llegando a la conclusión de que el uso extracto de Cranberry evita la adhesión de la placa dental a las piezas dentarias inferiores.

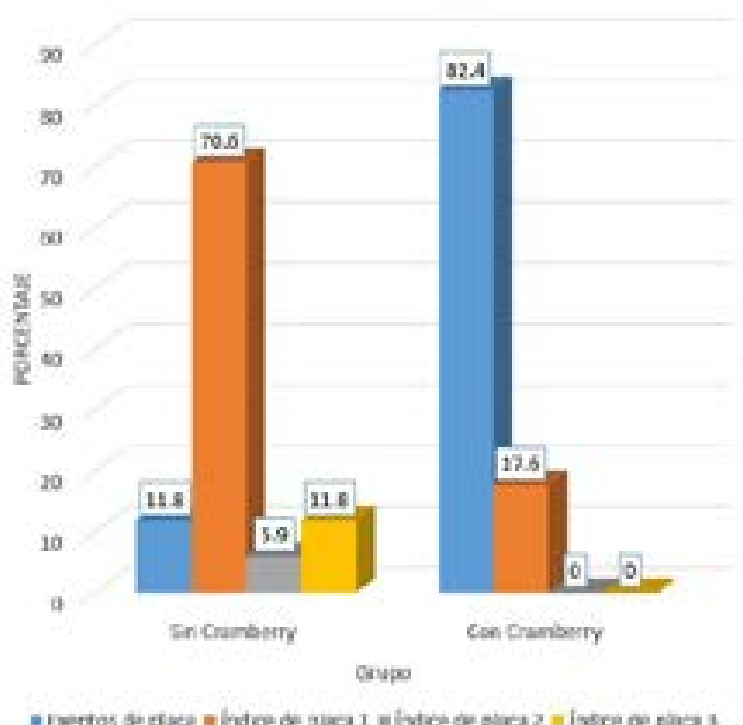

Gráfico 3: Comparación del índice de placa dental en la arcada inferior en los dientes de los alumnos antes y después del uso del extracto de Cranberry. Fuente: Matriz de datos

\section{DISCUSIÓN}

El estudio investigo el cranberry (vaccinium macrocarpon) y su efecto para evitar la adherencia de la placa bacteriana en las piezas dentales de los niños del colegio Horacio Morales de Arequipa Perú. El cranberry es rico en componentes que nos ayudan a combatir las enfermedades que más afectan a los niños de nuestro país como son las caries y las enfermedades periodontales. Chi Hua Wu 2007 demostró que el cranberry además de tener poder antiadherente también tiene poder antibacterial contra patógenos como E. Coli, L, monocyogenes y S. aureus es por esta razón que esta fruta está despertando el interés de muchos investigadores para poder descubrir los diferentes beneficios que puedan brindar para la salud de las personas.

El arándano es una fruta medicinal natural; se ha utilizado ampliamente como un suplemento dietético botánico popular, especialmente en forma de extracto de arándano, para prevenir y tratar las infecciones del tracto urinario causadas por UPEC (Escherichia coli uro patógena). Esta fruta es muy usada para combatir este tipo de infecciones urinarias por los diferentes compuestos que el cranberry posee. Analógicamente la misma película que se adhiere en las partes internas del tracto urinario también lo hace en la superficie del diente haciendo que las bacterias no colonicen en ninguna de las superficies de dichos órganos; evitando en el tracto urinario la infección y en el caso de las piezas dentales la desmineralización de los tejidos de los dientes.

Boteon 2017 en su estudio señala que las proantocianidinas presentes en el cranberry son capaces de reducir la degradación de la dentina validando nuestro estudio por la capacidad que tienen los cranberrys para evitar enfermedades en la boca.

Nuestra investigación corrobora la investigación de Vorsa donde se tomó un grupo de ratas a las cuales se les aplico el extracto de cranberry dos veces al día y presentaron notables cambios con respecto a las ratas que no recibieron el 
extracto en cuanto a adherencia de la placa en las superficies dentales de los dientes de los roedores. Coincidiendo con nuestro trabajo de investigación.

Vemos que, para prevenir la progresión de la enfermedad periodontal, dio como resultado que el uso de cranberry de alto peso molecular evitaba la colonización de las porphyromonas gingivalis por su efecto mecánico es decir evitaba que se adhiera favoreciendo la salud periodontal situación que puede revertir la salud gingival de pacientes con este riesgo. (Chandad, Daniel Grenier 2006) Dicha investigación va con el objetivo de la nuestra ya que nos demuestra el poder que tiene el cranberry para evitar que los microorganismos se adhieran a las superficies.

Es así como nuestra investigación arroja resultados alentadores con respecto al uso del cranberry en el ámbito odontológico por los múltiples efectos beneficiosos para la salud bucal.

Con el constante avance de la ciencia y los nuevos descubrimientos que se realizan día a día lograremos conocer todo el potencial que tienen los productos naturales para el beneficio de la salud de la población, para así poder ayudar a combatir las diversas enfermedades que aquejan a los seres humanos.

\section{CONCLUSIONES}

El índice de placa dental promedio en los dientes de niños antes de ser tratados con el extracto de Cranberry es 1.18 en nivel de placa dental en la arcada inferior y 1.71 en nivel de placa dental en la arcada superior.

El índice de placa dental promedio en los dientes de niños ya siendo tratados con el extracto de Cranberry es de 0.18 en nivel de placa dental en la arcada inferior y 0.29 en nivel de placa dental en la arcada superior.

Vemos que la diferencia de valores de placa dental de los días de estudio es muy significativa así que llegamos a la conclusión que usando el extracto de Cranberry reducimos mucho el nivel de placa dental adherida al esmalte de las piezas dentarias así evitamos caries y enfermedad periodontal.

\section{REFERENCIAS BIBLIOGRÁFICAS}

1. Mosaddad SA, Tahmasebi E, Yazdanian A, Rezvani MB, Seifalian A, Yazdanian M, et al. Oral microbial biofilms: an update. Eur J Clin Microbiol Infect Dis Off Publ Eur Soc Clin Microbiol. noviembre de 2019;38(11):2005-19.

2. Díaz-Cárdenas S, González-Martínez F. Prevalencia de caries dental y factores familiares en niños escolares de Cartagena de Indias, Colombia. Rev Salud Pública. octubre de 2010;12:843-51.

3. Pulido-Rozo M, Gonzalez-Martínez F, Rivas-Muñoz F. Enfermedad periodontal e indicadores de higiene bucal en estudiantes de secundaria Cartagena, Colombia. Rev Salud Pública. octubre de 2011;13:844-52.

4. Botero JE, Bedoya E. Determinantes del Diagnóstico Periodontal. Rev Clínica Periodoncia Implantol Rehabil Oral. 1 de agosto de 2010;3(2):94-9.

5. Pereyra JRH, López TT, Tello FJH, Moreno RR. Enfermedad periodontal: prevalencia y algunos factores asociados en escolares de una región mexicana. Rev Asoc Dent Mex. 2000;57(6):222-30.

6. Juárez-López MLA, Murrieta-Pruneda JF, TeodosioProcopio E. Prevalencia y factores de riesgo asociados a enfermedad periodontal en preescolares de la Ciudad de México. Gac Médica México. junio de 2005;141(3):185-9.

7. Martínez-Pabón MC, Morales-Uchima SM, MartínezDelgado CM. Caries dental en adultos jóvenes en relación con características microbiológicas y fisicoquímicas de la saliva. Rev Salud Pública. diciembre de 2013;15:844-56.

8. Garcés JCO, García EO, Salas LA. Streptococcus mutans y caries dental.(Streptococcus mutans and dental caries). CES Odontol. 16 de agosto de 2013;26(1):4456.

9. Cranberries_Effect_on_Biofilms_Chelius_Duffty.pdf [Internet]. [citado 30 de octubre de 2019]. Disponible en: https://web.wpi.edu/Pubs/E-project/Available/Eproject-041913-153923/unrestricted/Cranberries_ Effect_on_Biofilms_Chelius_Duffty.pdf

10. Antibacterial effects of American cranberry (Vaccinium macrocarpon) concentrate on foodborne pathogens ScienceDirect [Internet]. [citado 16 de noviembre de 2019]. Disponible en: https://www.sciencedirect.com/ science/article/pii/S0023643808000029

11. Kim SA, Kim HW, Rhee MS. Cranberry extract with enhanced bactericidal activities against uropathogenic Escherichia coli within one minute of treatment. LWT. 1 de octubre de 2019;113:108318.

12. Boteon AP, Kato MT, Buzalaf MAR, Prakki A, Wang L, Rios D, et al. Effect of Proanthocyanidin-enriched extracts on the inhibition of wear and degradation of dentin demineralized organic matrix. Arch Oral Biol. 1 de diciembre de 2017;84:118-24.

13. Labrecque J, Bodet C, Chandad F, Grenier D. Effects of a high-molecular-weight cranberry fraction on growth, biofilm formation and adherence of Porphyromonas gingivalis. J Antimicrob Chemother. 1 de agosto de 2006;58(2):439-43.

14. Koo H, Duarte S, Murata RM, Scott-Anne K, Gregoire S, Watson GE, et al. Influence of Cranberry Proanthocyanidins on Formation of Biofilms by Streptococcus mutans on Saliva-Coated Apatitic Surface and on Dental Caries Development in vivo. Caries Res. 2010;44(2):116-26. 